Racah Institute 151-Paris LPTHE 93-28

\title{
On the Calculability of Observables in Topological Quantum Mechanical Models
}

\author{
Laurent Baulieu周 \\ LPTHE, Université Pierre et Marie Curie, Paris \\ and \\ Eliezer Rabinovici门向 \\ Racah Institute of Physics, Hebrew University, Jerusalem
}

\begin{abstract}
We consider a superconformal quantum mechanical system which has been chosen on the basis of a local BRST topological invariance. We suggest that it truly leads to topological observables which we compute. The absences of a ground state and of a mass gap are special features of this system.
\end{abstract}

* Tour 16, 4 place Jussieu, 75005 Paris, France email address: Baulieu@lpthe.jussieu.fr

${ }^{\dagger}$ email address: Eliezer@vms.huji.ac.il

${ }_{\ddagger}$ Work supported by the Israeli Accademy of Sciences and the Bi-National American-Israeli Science Foundation 


\section{Introduction}

During the last years, Topological Quantum Field Theories have emerged as possible

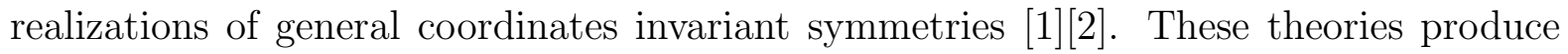
space-time metric independent correlations functions. For ordinary gauge theories, the well known phases are the massive Higgs phases and Coulomb and confinment phases. One of the great achievements of their study was the understanding that these phases have universality properties in the sense that their ulttraviolet behaviour are the same and that the short distance behaviour of the massive phase is milder than what one would expect for massive vector fields. It would be rewarding if Topological Quantum Field Theories, which have striking finitness properties at short distance, could be identified as a given phase of systems with an invariance under general reparametrization, so that the other phases, like quantum gravity, would also reveal finitess properties.

In ordinary gauge theories the study of the behaviour of order parameters and relevant operators has been a usefull tool to survey the possible phases. The interesting question of finding the relevant order parameters for general ccordinate invariant systems is yet unresolved.

In Topological Quantum Field Theories, an important operator which is at disposal is the BRST operator $Q$, such that the Hamiltonian is $H=\frac{1}{2}[Q, \bar{Q}]$. One usually defines the physical Hilbert space as the cohomolgy of $Q$ (states which are annihilated by $Q$ without being the $Q$ transformation of other states). This definition of the physical Hilbert space is perfectly suited for ordinary gauge theories. For Topological Field Theories there are doubts on the validity of this definition and their could be other relevant observables than those defined by the BRST cohomology. One may think that the failure of finding states annihilated by $Q$ but the $Q$ exact ones would signal the transition to the topological phase. In supersymmetric theories a similar situation indeed implies the breaking of supersymmetry and would make possible the relevance of $Q$ exact observables. Actually the absence of states annihilated by the constraints is sometimes the symptom of a global gauge anomaly. It may also occur that a more refined form ofthe BRST symmetry should be introduced, the cohomology of which would contain the $Q$ exact observables. 
In the Topological Quantum Field Theories such as those originally introduced in [1] which can be understood as the gauge fixing of topological invariants [4], the observables can be seen to be $Q$ exact. In the other topological models based on first order actions like the Chern-Simons action [3], formal argument show that the situation is similar. In all these models one sees furthermore that, in addition to the global topological BRST symmetry, a local version of it seems to characterize the supersymmetric potential [5] [6]. However, little is known about how the supersymmetry and ghost number conservation laws are truly broken to yield non vanishing mean values of topological observables, and also how one computes these mean values.

In this note we wish to study these questions in the framework of a simplest model which shares the characteristics of the more sophisticated models based on the gauge fixing of topological invariants. This model is a supersymmetric quantum mechanical system defined on a punctured plane. It has been chosen under the condition that it has an additional quasi-local symmetry. This symmetry turns out to be quite similar to a conformal supersymmetry and thus we can use technics already introduced in [7]. It is known that by a Wick rotation any supersymmetric quantum mechanics action can be understood as a topological action [2] and moreover the potential of our model is based on the criteria of a local BRST invariance which we believe warrantees its non triviality [5]. This model is solvable, and we verify that due to the superconformal properties of its potential it has some rather special features that we study in details. The spectrum is continuous and contains all $E>0$ states but not the state with energy 0 which is not even plane wave normalizable. Thus supersymmetry is broken. Let us recall that Witten suggested that non normalizability of the wave function could be a general criteria for the signal of a phase transition [8]. We then verify the existence of $Q$ exact topological observable of which we compute the mean values. Moreover, we notice that this groundstateless system possesses an interesting instanton structure and that all states are infinitely degenerate with respect to the discrete angular momentum quantum number. It seems that the answer that we obtain for topological observables means that in the path integral one gets one single contribution from each each instanton sector. The observable that we get is simply related to a generator of a superconformal 
algebra. We have also computed the Witten index and observed that what contributes to its value is the non normalizable and measure zero part of the spectrum. As far as the exploration of the model is concerned we have adopted an open attitude: we could have rejected it as having a global anomaly, but we didn't and attempted to extract from it scale independant features.

\section{The Model}

We wish to work with a simple topological classical Lagrangian that is a candidate to generate a topological quantum mechanics. We consider as a target space a plane from which we exclude the origin, so that one has a non trivial, although very simple topological structure defined by the winding number around the origin of the trajectories of a particle. We denote the time by the real variable $t$ and the Euclidian time by $\tau$, with $t=i \tau$ and $\tau$ real. The cartesian coordinates on the plane are $q_{i}$, with $i=1,2$. We select trajectories with periodic conditions, namely such that between the initial and final times $t=0$ and $t=T$ the particle ends up at its starting point so an integer value of the winding number can be assigned to its trajectory.

From our understanding of the nature of a topological field theory [4], we start from a topological classical action $\mathcal{I}_{c l}[\vec{q}]$. $\mathcal{I}_{c l}[\vec{q}]$ must not depend on the time metric. This condition is satisfied if it is the integral of a locally closed form. The natural candidate is

$$
\begin{aligned}
\mathcal{I}_{c l}[\vec{q}]=\int f d \theta & =\int_{0}^{T} d \tau f \dot{\theta}(\tau) \\
& =\int_{0}^{T} d \tau f \frac{\epsilon^{i j} \dot{q}_{i} q_{j}}{\vec{q}^{2}}
\end{aligned}
$$

where $f$ is a real number. This action measures the winding number of the particle times $f / 2 \pi$. It shares analogy with the second Chern class $\int d^{4} x \operatorname{tr} F \wedge F$ where $F$ is the curvature of a Yang-Mills field. Here and in what follows the symbol $\dot{X}$ denotes $\frac{d X}{d \tau}$.

To obtain the Topological Quantum Theory associated to our space, we need to give 
sense to the Euclidian path integral

$$
\int \mathcal{D}[\vec{q}] \exp -\mathcal{I}_{c l}[\vec{q}]
$$

as well as to compute topological quantities from Green functions

$$
\text { Topological information }=\int \mathcal{D}[\vec{q}] O \exp -\mathcal{I}_{c l}[\vec{q}]
$$

where $O$ is a well chosen composite operator.

The difficulty for realizing this objective is that our action is different from that of conventional quantum mechanics where classical degrees of freeedom exist at the classical level and quantum fluctuations occur around the solutions of equations of motion. Here the Lagrangian is locally a pure derivative, the Hamiltonian vanishes and one has no equation of motion. On the other hand, one observes that the action $\mathcal{I}_{c l}[\vec{q}]$ is invariant under the gauge symmetry

$$
q(\overrightarrow{t)} \rightarrow q \overrightarrow{(t)}+\epsilon \overrightarrow{(t)}
$$

where $\epsilon(t)$ is any given local shift of the particle position $q(t)$ which does not change the winding number of the trajectory. Using the BRST technique it is then possible to define the path integrals $(2.2)$ and $(2.3)$ by a conventional gauge fixing of the action $\mathcal{I}_{c l}[\vec{q}]$.

The BRST transformation laws associated to the symmetry (2.4) are of the simple form

$$
s \vec{q}=\vec{\Psi} \quad s \vec{\Psi}=0 \quad s \vec{\Psi}=\vec{\lambda} \quad s \vec{\lambda}=0
$$

The anticommuting fields $\vec{\Psi}(t)$ and $\vec{\Psi}(t)$ are the topological ghosts and antighosts associated to the particle position $\vec{q}(t) . \vec{\lambda}(t)$ is a Lagrange multiplier. s acts on field functions as a differential operator graded by the ghost number.

To get a gauge fixed action with a quadratic dependence on the velocity $\overrightarrow{\dot{q}}$, one choses a gauge function of the type $\dot{q}_{i}+\frac{\delta V}{\delta q_{i}}$, where the prepotential $V$ is an arbitrary given function of $\vec{q}$. This yields the following gauge fixed BRST invariant action $\mathcal{I}_{g f}$ which is supersymmetric

$$
\mathcal{I}_{g f}[\vec{q}, \vec{\Psi}, \vec{\Psi}, \vec{\lambda}]=\int_{0}^{T} d \tau\left(f \dot{\theta}-s \bar{\Psi}_{i}\left(\frac{1}{2} \lambda_{i}-i \dot{q}_{i}+\frac{\delta V}{\delta q_{i}}\right)\right)
$$




$$
=\int_{0}^{T} d \tau\left(f \dot{\theta}-\frac{1}{2} \lambda_{i}^{2}+i \lambda_{i}\left(\dot{q}_{i}+\frac{\delta V}{\delta q_{i}}\right)-i \bar{\Psi}_{i}\left(\dot{\Psi}_{i}+\frac{\delta^{2} V}{\delta q_{i} \delta q_{j}} \Psi_{j}\right)\right)
$$

The BRST symmetry $s \mathcal{I}_{g f}[\vec{q}, \vec{\Psi}, \vec{\Psi}, \vec{\lambda}]=0$ holds true independently of the choice of the function $V(\vec{q})$ and the partition function and the mean values of BRST invariant observables

$$
\begin{gathered}
Z=\int \mathcal{D}[\vec{q}] \mathcal{D}[\vec{\Psi}] \mathcal{D}[\vec{\Psi}] \mathcal{D}[\vec{\lambda}] \exp -\mathcal{I}_{g f} \\
<O>=\int \mathcal{D}[\vec{q}] \mathcal{D}[\vec{\Psi}] \mathcal{D}[\vec{\Psi}] \mathcal{D}[\vec{\lambda}] O \exp -\mathcal{I}_{g f}
\end{gathered}
$$

are now well defined Euclidian path integrals. To understand $\dot{q}_{i}+\frac{\delta V}{\delta q_{i}}$ as a gauge function for the quantum variable $\vec{q}$, one may interpret the result of the integration over the ghosts as a determinant. The BRST invariance of the field polynomial $O$ allows one to prove, at least formally, the topological properties of $\langle O\rangle$. On the other hand our knowledge of supersymmetric quantum mechanics tells us that this mean value may depend on the class of the function $V$. What happens is that in the case of topological field theories, the Euclidian path integral explores the moduli space of the equation $\dot{q}_{i}+\frac{\delta V}{\delta q_{i}}=0$, as a result of the gauge fixing.

The question of finding a symmetry principle which would select the prepotential $V(\vec{q})$ leading to interesting topological information was investigated in $[5]$. The idea is to ask for the invariance of the action under a symmetry which is more restrictive than the topologocal BRST symmetry, namely a local version of it, for which the parameter becomes an affine function of the time, with arbitrary infinitesimal coefficients. One requires

$$
\delta_{l} \mathcal{I}_{g f}[\vec{q}, \vec{\Psi}, \vec{\Psi}, \vec{\lambda}]=0
$$

where the "local" BRST transformations $\delta_{l}$ are

$$
\delta l \vec{q}=\eta(t) \vec{\Psi} \quad \delta_{l} \vec{\Psi}=0 \quad \delta_{l} \vec{\Psi}=\eta(t) \vec{\lambda}-\dot{\eta}(t) \vec{q} \quad \delta_{l} \vec{\lambda}=\dot{\eta}(t) \vec{\Psi}
$$

and $\eta(t)=a+b t$ where $a$ and $b$ are constant anticommuting parameters. The idea of local BRST symmetry was considered in [9] for the sake of interpreting higher order cocycles 
which occurs when solving the anomaly consistency conditions, and has been shown to play a role in topological field theories in [6].

Imposing this local symmetry implies that $V$ satisfies the constraint [5]

$$
\frac{\delta V}{\delta q_{i}}+q_{j} \frac{\delta^{2} V}{\delta q_{i} \delta q_{j}}=0
$$

This constraint is solved for $V(\vec{q})=f \theta$ where $\theta$ is the angle such that $q_{1}+i q_{2}=|\vec{q}| \exp i \theta$ and $\mathrm{f}$ is a number [5]. By putting this value of $\dot{q}_{i}+\frac{\delta V}{\delta q_{i}}$ in (2.6) and eliminating the Lagrange multiplier $\lambda$ by its equation of motion we obtain

$$
\mathcal{I}_{g f}[\vec{q}, \vec{\Psi}, \vec{\Psi}]=\int_{0}^{T} d \tau\left(\frac{1}{2} \dot{q}_{i}^{2}+\frac{f^{2}}{2 \vec{q}^{2}}-\bar{\Psi}_{i}\left(\dot{\Psi}_{i}+f \frac{\delta^{2} \theta}{\delta q_{i} \delta q_{j}} \Psi_{j}\right)\right)
$$

Notice that

$$
\begin{aligned}
\frac{\delta^{2} \theta}{\delta q_{i} \delta q_{j}} & =\frac{1}{\vec{q}^{2}}\left(\begin{array}{cc}
-\sin 2 \theta & \cos 2 \theta \\
\cos 2 \theta & \sin 2 \theta
\end{array}\right)_{i j} \\
& =\frac{1}{\vec{q}^{2}}\left(\begin{array}{cc}
\cos \theta & -\sin \theta \\
\sin \theta & \cos \theta
\end{array}\right)\left(\begin{array}{cc}
0 & -1 \\
-1 & 0
\end{array}\right)\left(\begin{array}{cc}
\cos \theta & \sin \theta \\
-\sin \theta & \cos \theta
\end{array}\right)_{i j}
\end{aligned}
$$

The superconformal potential $1 / \vec{q}^{2}$ has been already studied in [7] [10. In the next section we shall compute the observables which seems interesting to us from the topological point of view in the canonical quantization formalism. We will show that a very specific supersymmetry breaking mechanism occurs and implies the existence of non vanishing $Q$ exact observables which are metric independent as well as of a fractional Witten indexD.

We believe that the signal that the theory truly carries some topological information is the existence of an interesting instanton structure. Let us remember that, from our gauge fixing in the Euclidian time region, we have obtained an action whose bosonic part is the square of the gauge function. It follows that the solutions to the Euclidien equations of motion can be written as

$$
\dot{q}_{i} \pm f \frac{\epsilon^{i j} q_{j}}{\vec{q}^{2}}=0
$$

\footnotetext{
${ }^{1}$ For the case of one variable $x$ we would obtain $V=\log x$, with quite similar properties of the supersymmetric system, but the geometrical interpretation would be less clear and no meaningful observable exists
} 


$$
\dot{\Psi}_{i} \pm f \frac{\delta^{2} \theta}{\delta q_{i} \delta q_{j}} \Psi_{j}=0
$$

If we introduce $z=q_{1}+i q_{2}$ and $\Psi_{z}=\Psi_{1}+i \Psi_{2}$, with $s z=\Psi_{z}$, we can write these equations as

$$
\begin{gathered}
\dot{z}+\frac{i}{z^{*}}=0 \\
\dot{\Psi}_{z}-\frac{i}{\left(z^{*}\right)^{2}} \Psi_{z}^{*}=0
\end{gathered}
$$

Assuming periodic boundary conditions, the solutions for $\vec{q}$ are circles described at constant velocities and indexed by an integer $n$

$$
z^{(n)}=\sqrt{\frac{T}{2 n \pi}} \exp -i \frac{2 n t}{T} \quad n \in Z
$$

while for the ghost

$$
\Psi_{z}^{(n)}=\eta \exp i \frac{2 n t}{T}
$$

where $\eta$ is a constant fermion. The Euclidian energy and angular momentum of the action evalueted for these field configurations vanish for all valuese of $n$.

Due to the existence of these degenerate zero modes of the action we expect that BRST invariant observables should exist and that their mean values should be non zero as well as energy and time reparametrization independent. The corresponding numbers should to be expressable as a series over an integer related to the one which label the instanton solutions. This is the conjecture that we shall verify in the next section.

\section{Hamiltonian Quantization}

When possible, it is worthwhile to compute observables in the canonical formalism. We do a Wick rotation to recover the real Minkowski time $t$ by setting $\tau=i t$, and change the quantum mechanichal variables into operators. The Hamiltonian associated to the action $\mathcal{I}_{g f}$ is

$$
H=\frac{1}{2} \vec{p}^{2}+\frac{f^{2}}{2 \vec{q}^{2}}-f \bar{\Psi}_{i} \frac{\delta^{2} \theta}{\delta q_{i} \delta q_{j}} \Psi_{j}
$$


where the quantization rules are ( remember that $q_{i}=(x, y)$ stands for the cartesian coordinates on the plane)

$$
\begin{gathered}
{\left[p_{i}, q_{j}\right]=-i \delta_{i j} \quad\left[\bar{\Psi}_{i}, \Psi_{j}\right]_{+}=\delta_{i j}} \\
{\left[\Psi_{i}, \Psi_{j}\right]_{+}=\left[\bar{\Psi}_{i}, \bar{\Psi}_{j}\right]_{+}=\left[\Psi_{i}, p_{j}\right]=\left[\bar{\Psi}_{i}, p_{j}\right]=\left[\Psi_{i}, q_{j}\right]=\left[\bar{\Psi}_{i}, q_{j}\right]=0}
\end{gathered}
$$

By construction $H$ can be written as

$$
H=\frac{1}{2}\{Q, \bar{Q}\}
$$

with

$$
Q=\Psi_{i}\left(p_{i}+i f \frac{\delta \theta}{\delta q_{i}}\right) \quad \bar{Q}=\Psi_{i}\left(p_{i}-i f \frac{\delta \theta}{\delta q_{i}}\right)
$$

Following [10], we use the following matricial representation for the ghost and antighost operator

$$
\Psi_{1}=\left(\begin{array}{cccc}
0 & 1 & 0 & 0 \\
0 & 0 & 0 & 0 \\
0 & 0 & 0 & 1 \\
0 & 0 & 0 & 0
\end{array}\right) \quad \Psi_{2}=\left(\begin{array}{cccc}
0 & 0 & -1 & 0 \\
0 & 0 & 0 & 1 \\
0 & 0 & 0 & 0 \\
0 & 0 & 0 & 0
\end{array}\right)
$$

One has $\bar{\Psi}=\Psi^{\dagger}$ and $p_{i}=-i \delta / \delta q_{i}$. In this representation

$$
H=\left(\begin{array}{cccc}
H_{0} & 0 & 0 & 0 \\
0 & H_{11} & H_{12} & 0 \\
0 & H_{21} & H_{22} & 0 \\
0 & 0 & 0 & H_{2}
\end{array}\right)
$$

where

$$
H_{0}=H_{2}=-\frac{1}{2 r} \frac{\delta}{\delta r} r \frac{\delta}{\delta r}-\frac{1}{2 r^{2}} \frac{\delta^{2}}{\delta \theta^{2}}+\frac{f^{2}}{2 r^{2}}
$$

and

$$
\begin{aligned}
\left(\begin{array}{ll}
H_{11} & H_{12} \\
H_{21} & H_{22}
\end{array}\right) & =H_{0} \delta_{i j}+f \frac{\delta^{2} \theta}{\delta q_{i} \delta q_{j}} \\
& =R_{-\theta}\left(-\frac{1}{2 r} \frac{\delta}{\delta r} r \frac{\delta}{\delta r}+\frac{f^{2}+1-\frac{\delta^{2}}{\delta \theta^{2}}}{2 r^{2}}-\frac{1}{r^{2}}\left(\begin{array}{cc}
0 & f+i \frac{\delta}{\delta \theta} \\
f-i \frac{\delta}{\delta \theta} \theta & 0
\end{array}\right)\right) R_{\theta}
\end{aligned}
$$


where $r$ and $\theta$ are the polar coordinates on the plane and

$$
R_{\theta}=\left(\begin{array}{cc}
\cos \theta & \sin \theta \\
-\sin \theta & \cos \theta
\end{array}\right)
$$

The spectrum of $H$ is straightforward to derive in this representation. One uses the usual strategy based on the fact that if an eigenstate of $H$ has energy $E$, its $Q$ and $\bar{Q}$ transforms are either zero or an eigenstate of $H$ with the same energy. States are labelled by their non negative energy $E$, angular momentum $\mathrm{n}$ and fermion number $\alpha$, that is ghost number. We denote them as $|E, n, \alpha\rangle$. For each value $E$ and $n$, one has four states labelled by $\alpha=1,2,3,4$. The states with $\alpha=1$ and $\alpha=4$ are respectively anihilated by $Q$ and $\bar{Q}$. This is due to the fact that states $\mid \phi>$ which are BRST invariant, $Q \mid \phi>=0$, are such that

$$
\begin{gathered}
\left|\phi>=\left(\begin{array}{c}
\mid E, n> \\
0 \\
0 \\
0
\end{array}\right) \quad \bar{Q}\right| \phi>=\left(\begin{array}{c}
0 \\
\left(p_{1}-i f \frac{\delta \theta}{\delta q_{1}}\right) \mid E, n> \\
-\left(p_{2}-i f \frac{\delta \theta}{q_{2}}\right) \mid E, n> \\
0
\end{array}\right) \\
H \mid \phi>=\left(\begin{array}{c}
H_{0} \mid E, n> \\
0 \\
0 \\
0
\end{array}\right)
\end{gathered}
$$

One has similar relations for states $\mid \bar{\phi}>$ satisfying $\bar{Q} \mid \bar{\phi}>=0$.

Let us define $g_{E, n}=<r, \theta \mid E, n>$. This function is the solution of the equation

$$
<r, \theta\left|H_{0}\right| E, n>=\left(-\frac{1}{2 r} \frac{\delta}{\delta r} r \frac{\delta}{\delta r}-\frac{1}{2 r^{2}} \frac{\delta^{2}}{\delta \theta^{2}}+\frac{f^{2}}{2 r^{2}}\right) g_{E, n}=E g_{E, n}
$$

$g_{E, n}$ is also the solution of the ghost number 2 equation $\left\langle r, \theta\left|H_{2}\right| E, n>=E\right| E, n>$. Its knowledge is sufficient to get the full spectrum for $E \neq 0$. One has indeed

$$
\left|E, n, 1>=\left(\begin{array}{c}
\mid E, n> \\
0 \\
0 \\
0
\end{array}\right) \quad\right| E, n, 2>=\frac{1}{\sqrt{E}} \bar{Q} \mid E, n, 1>
$$




$$
\left|E, n, 4>=\left(\begin{array}{c}
0 \\
0 \\
0 \\
\mid E, n>
\end{array}\right) \quad\right| E, n, 3>=\frac{1}{\sqrt{E}} \bar{Q} \mid E, n, 4>
$$

The diagonalization of the part with ghost number one of the Hamiltonian (3.8) amounts to solve the equations

$$
\left(-\frac{1}{2 r} \frac{\delta}{\delta r} r \frac{\delta}{\delta r}+\frac{f^{2}+1-\frac{\delta^{2}}{\delta \theta^{2}} \pm 2 \sqrt{f^{2}-\frac{\delta^{2}}{\delta \theta^{2}}}}{2 r^{2}}\right) g_{E, n, \pm}=E g_{E, n, \pm}
$$

which are of the same type as (3.11).

To solve (3.11) and (3.13) we set

$$
\begin{aligned}
g_{E, n}=\frac{1}{\sqrt{2 \pi}} \exp i n \theta f_{E, n}(r) \quad n \in Z \\
g_{E, n, \pm}=\frac{1}{\sqrt{2 \pi}} \exp i n \theta f_{E, n, \pm}(r) \quad n \in Z
\end{aligned}
$$

For $E \neq 0, f_{E, n}(r)$ and $f_{E, n, \pm}$ are expressable as a Bessel function $J_{\nu}(\sqrt{2} E r)$ of order $\nu$, with

$$
f_{E, n}(r)=\frac{1}{\sqrt{2}} J \sqrt{n^{2}+f^{2}}(\sqrt{2} E r)
$$

and

$$
f_{E, n, \pm}(r)=\frac{1}{\sqrt{2}} \exp i n \theta J \sqrt{f^{2}+1+n^{2} \pm 2 \sqrt{f^{2}+n^{2}}}(\sqrt{2} E r)
$$

These states are normalizable as plane waves in one dimension. This is a consequence of the continuity of the spectrum in the radial direction. They build an appropriate basis of stationary solutions since, with the normalization factor which is explicit in (3.15), one has $\sum_{n} \int_{E>0} d E|E, n><E, n|=1$. On the other hand, for $E=0$, the Schrödinger equations (3.11) and (3.13) have no admissible normalizable solution. Thus we have a continuum spectrum, bounded from below, with a spin degeneracy equal to 4 and an infinite degeneracy in the angular momentum quantum number $n$. The peculiarity of this spectrum is that there is no ground state, since we have states with energy as little 
as we want, but we cannot have $E=0$. This is a consequence of the conformal property of the potential $\frac{1}{|\vec{q}|^{2}}$.

Since we cannot reach the energy zero which woud be the only $Q$ and $\bar{Q}$ invariant state, we conclude that supersymmetry is broken.

It is useful for what follows to redefine the ghost and antighost operators into

$$
\left(\begin{array}{c}
\Psi_{r} \\
\Psi_{\theta}
\end{array}\right)=\left(\begin{array}{cc}
\cos \theta & \sin \theta \\
-\sin \theta & \cos \theta
\end{array}\right)\left(\begin{array}{l}
\Psi_{1} \\
\Psi_{2}
\end{array}\right) \quad\left(\begin{array}{l}
\bar{\Psi}_{r} \\
\bar{\Psi}_{\theta}
\end{array}\right)=\left(\begin{array}{cc}
\cos \theta & \sin \theta \\
-\sin \theta & \cos \theta
\end{array}\right)\left(\begin{array}{l}
\bar{\Psi}_{1} \\
\bar{\Psi}_{2}
\end{array}\right)
$$

These rotated ghost operators satisfy similar anticommutation rotations as the $\Psi_{i}$ and $\bar{\Psi}_{i}$. On the other hand, notice that

$$
\begin{gathered}
{\left[\frac{\delta}{\delta \theta},\left(\begin{array}{c}
\bar{\Psi}_{r} \\
\bar{\Psi}_{\theta}
\end{array}\right)\right]_{+}=\left(\begin{array}{c}
\bar{\Psi}_{\theta} \\
-\bar{\Psi}_{r}
\end{array}\right) \quad\left[\frac{\delta}{\delta \theta},\left(\begin{array}{c}
\Psi_{r} \\
\Psi_{\theta}
\end{array}\right)\right]=\left(\begin{array}{c}
\Psi_{\theta} \\
-\Psi_{r}
\end{array}\right)} \\
{\left[\frac{\delta}{\delta r},\left(\begin{array}{c}
\bar{\Psi}_{r} \\
\bar{\Psi}_{\theta}
\end{array}\right)\right]=\left[\frac{\delta}{\delta r},\left(\begin{array}{c}
\Psi_{r} \\
\Psi_{\theta}
\end{array}\right)\right]_{+}=0}
\end{gathered}
$$

One has the following expression of $Q$ and $\bar{Q}$ which will be used in the next section

$$
Q=-i \Psi_{r} \frac{\delta}{\delta r}-i \frac{1}{r} \Psi_{\theta}\left(\frac{\delta}{\delta \theta}-f\right) \quad \bar{Q}=-i \bar{\Psi}_{r} \frac{\delta}{\delta r}-i \frac{1}{r} \bar{\Psi}_{\theta}\left(\frac{\delta}{\delta \theta}+f\right)
$$

These expressions in curved coordinates could be obtained from the general formalism of [11].

\section{Computation of BRST Invariant Observables}

In the last section we have seen that supersymmetry is broken in a very special way. This opens the possibility of having non vanishing BRST-exact Green functions which are topological in the sense that they are scale independent, that is independent of time, or energy, rescalings.

From dimensional arguments the candidates for such commutators are

$$
O_{\theta}=\left[Q, r \bar{\Psi}_{\theta}\right]_{+}=\left[\bar{Q}, r \Psi_{\theta}\right]_{+}^{\dagger} \quad O_{r}=\left[Q, r \bar{\Psi}_{r}\right]_{+}=\left[\bar{Q}, r \Psi_{r}\right]_{+}^{\dagger}
$$


The mean values of these operators between normalized states are

$$
\frac{<E, n\left|\left[Q, r \bar{\Psi}_{\theta}\right]_{+}\right| E, n>}{<E, n \mid E, n>}=n+i f
$$

and

$$
\frac{<E, n\left|\left[Q, r \bar{\Psi}_{r}\right]_{+}\right| E, n>}{<E, n \mid E, n>}=\lim _{L \rightarrow \infty} \frac{L^{2} J^{2} \sqrt{n^{2}+f^{2}}(L)}{\int_{0}^{L} d r J \sqrt{n^{2}+f^{2}}(r)}
$$

The last quantity is bounded but ill-defined, so we reject it. We get therefore that for any normalized state $\left|\phi_{n}\right\rangle=\int d E \rho(E) \mid E, n>$ with a given angular momentum $n$, the expectation value of $\left[Q, r \bar{\Psi}_{\theta}\right]_{+}$is

$$
<\phi_{n}\left|\left[Q, r \bar{\Psi}_{\theta}\right]_{+}\right| \phi_{n}>=n+i f
$$

indepently of the weighting function $\rho$.

If we now sum over all values of $n$, what remains is the topological number

$$
<O_{\theta}>=\sum_{n}<\phi_{n}\left|\left[Q, r \bar{\Psi}_{\theta}\right]_{+}\right| \phi_{n}>=\sum_{n} n+i f \sum_{n} 1
$$

From a topological point of view, our result mean that there are two observables, organized in a complex form, in the cohomology of the punctured plane. The summation over the index $n$, that is the angular momentum, could have expected from the formal argument that in the path integral one gets a single finite contribution from each instanton solution to the mean value of a topological observable, so that

$$
\text { Topological information }=\int \mathcal{D}[\vec{q}] O_{f} \exp -\mathcal{I}_{c l}[\vec{q}] \sim \sum_{n} f(n)
$$

Our computation shows the existence of a BRST invariant observable with non zero mean value which is $Q$-closed. The supersymmetry breaking mechanism made possible by our potential choice (on the basis of local BRST symmetry) is responsible of this situation. With other potentials than the one that we have chosen, either supersymmetry would be unbroken, or a mass gap would occur. In the previous case all Q-exact observable would vanish; in the latter case they could be nonzero but they would be scale dependent.

As another topological observable of the theory, we may consider the Witten index 12] [13]. The idea is that although there is no normalizable vacuum in the theory, we 
can consider the trace

$$
\Delta=\operatorname{Tr}(-)^{F} \exp -\beta H
$$

where the trace means a sum over angular momentum as well as over all energy including energy zero, and $(-)^{F}$ is the ghost or fermion number operator. The result should be finite because, although the state with energy zero is not normalizable, it contributes only over a domain of integration with zero measure. Indeed, since supersymmetric compensations occur for $E \neq 0$ and provided one uses a BRST symmetry preserving regularization, the full contribition to $\Delta$ should come from the domain of integration concentrated at $E \sim 0$, while the topological nature of the theory should warranty that $\Delta$ is non zero and independent on $\beta$.

By using the suitably normalized eigenfunctions of the Hamiltonian, eqs.(3.15) and (3.16), one can write the index $\Delta$ as follows

$$
\begin{aligned}
& \Delta=\sum_{n} \int_{0}^{\infty} d E \quad \exp \quad-\beta E \int r d r \frac{1}{2}\left(2 J_{\sqrt{n^{2}+f^{2}}}(\sqrt{2 E} r)\right. \\
& \left.-\quad J^{2} \sqrt{f^{2}+1+n^{2}+\sqrt{f^{2}+n^{2}}}(\sqrt{2 E} r)-J^{2} \sqrt{f^{2}+1+n^{2}-\sqrt{f^{2}+n^{2}}}(\sqrt{2 E} r)\right)
\end{aligned}
$$

To compute this double integral one needs a regularisation. Following for instance [13], we can use a dimensional regularization. Thus we change $d r$ into $r^{\epsilon} d r$. Then, the analytic comtinuation of the result when $\epsilon \rightarrow 0$ is

$$
\Delta=\sum_{n} \frac{1}{2}\left(2 \sqrt{f^{2}+n^{2}}-\sqrt{f^{2}+1+n^{2}+\sqrt{f^{2}+n^{2}}}-\sqrt{f^{2}+1+n^{2}-\sqrt{f^{2}+n^{2}}}\right)
$$

As announced this result is independent on $\beta$. As a series, it diverges logarimically as $\sum 1 / n$ which is presumably the consequence of the conformal invariance of the potential. We see that the contribution of each topological sector is $n$ dependant. 


\section{$5 \quad$ Underlying superconformal properties}

We now explain our result of getting energy independant expectation value of a Q-exact operator as a consequence of the underlying superconformal structure of our model. Firstly, let us investigate the possible consequences of having an operator $A$ which commutes with the Hamiltonian $H$ and which is BRST exact, that is $A=[Q, X]$. A has zero expectation values between physical states, that is $Q$ and $\bar{Q}$ invariant states. For non physical states, that is states of given non zero energy E, each eigen-value of the operator $A$ is a priori implicitely dependent on the value of $E$, since although $A$ and $H$ are simultaneously diagonalizable, their eigenvalues are generally connected. However, it may happen that this dependance is eliminated if, for each value of $E$, all possible eigenvalues of $A$ are allowed. This is exactly the situation which occurs in our model where the observable shown in (4.2) is of the type of such an operator $A$ and where one can verify that for all values of $E$ all possible values of the spin and the angular momentum are allowed for. We can can therefore consider the summation shown in (4.5), or the limit of (4.2) when $E \rightarrow 0$.

The existence of this observable could have been foreseen from the superconformal properties of the potential. Consider first the case where we would be in one dimension $x$, with the same potential in $f / q^{2}$. In this case the candidate for a topological observable doesnot lead to a well defined result. To prove this, following [7], we define the following superconformal algebra generators

$$
S=\Psi x \quad \bar{S}=\bar{\Psi} x \quad K=\frac{1}{2} x^{2}
$$

One has the superconformal algebra relations

$$
\begin{gathered}
\frac{1}{2}[\bar{S}, S]_{+}=K \\
\frac{1}{2}[Q, \bar{S}]_{+}=\frac{f}{2}-\frac{1}{4}[\bar{\Psi}, \Psi]_{+}-i D
\end{gathered}
$$

where $D$ is dilatation operator

$$
D=-\frac{1}{4}[x, p]_{+}
$$


One can verify that $D$ does not commute with $H$,

$$
l D, H]=-i H
$$

which means that in this case the operator $A=\frac{1}{2}[Q, \bar{S}]_{+}$is not useful to obtain $E$ independant observables. What happen is that $\langle D\rangle$ leads to an undefinned result, similar to (4.3), with $r$ replaced by the one dimensional variable $x$ and no well defined observable exists in this situation.

Consider now our case with two dimensions. One defines

$$
S=\Psi_{i} x^{i} \quad \bar{S}=\bar{\Psi}_{i} x^{i}
$$

Due to the the $O(2)$ invariance one also defines

$$
S^{\prime}=\epsilon_{i j} \Psi_{i} x^{j} \quad \bar{S}^{\prime}=\epsilon_{i j} \bar{\Psi}_{i} x^{j}
$$

One can then verify that, independently of the value of the coupling $f$

$$
\left[Q, \bar{S}^{\prime}\right]_{+}=i J \quad[H, J]=0
$$

It follows that the operator $J$, which is part of the superconformal algebra of quantum mechanics in two dinensions, can be used as the observable $A$ discussed at the beginning of this section. More precisely, to interpret the obervables written in (4.4), one can one identify the operator $J$ and the eigenvalues $\sum n$, and the identity operator with the eigenvalues $f \sum 1$.

We conclude this section by recalling that a way to regulate conformal supersymmetric quantum mechanics and allow for the existence of a ground state was proposed in [7]. It was suggested to change the time evolution generator, that is the Hamiltonian $H$ into

$$
H=\frac{1}{2}\left(a H+a^{-1} K\right)
$$

As a result, the BRST operator is changed by terms of order $a^{-1}$, a normalisable ground state occurs for which the mean values of our observables vanish. What is interesting is that the eigenstates of the new time evolution operator, when expressed in terms of those of $H$ build a spectrum not dissimilar to that involved in blackhole physics [7]. 


\section{6 discussion}

We have shown an example for which the requirement of local BRST symmetry for topological quantum mechanics results in selecting a superconformal quantum mechanichal system. As a result, the spectrum of the theory has no ground state and a supersymmetry breaking mechanism occurs, without the the presence of a dimensionful parameter. Our goal was to understand the mechanism which provide topological observables. We observed that the special properties of the potential allows the computation of energy independant quantities although they are of mean values of BRST exact observables between non zero energy states. These quantities deserve to be called topological and they get a contribution from the whole spectrum of the theory. We have also singled out the Witten index, in a computation which includes a contribution from the non normalizable state of zero energy. The generalization of these observations to quantum field theory is an interesting open question.

We wish to thank T. Banks and S. Elitzur for very useful discussions. 


\section{References}

[1] E. Witten, Comm. of Math. Phys. 117, (1988), 353; Comm. of Math. Phys. 118, (1988) 601; Phys. Lett. B206 , 1988.

[2] For a review see O. Birmingham, M. Blau, M. Rakowski and G. Thomson, Physics Reports 209, (1991), 129 and references therein.

[3] E. Witten, Comm. of Math. Phys. 121, (1989), 351.

[4] L. Baulieu and I.M. Singer, Nucl. Phys. Proc. Suppl. 5B, (1988), 12; Comm. of Math. Phys. 125, (1989), 227; Comm. of Math. Phys. 135, (1991), 253.

[5] L. Baulieu and C. Aragao de Carvalho Phys. Lett. B275 (1991)323; Phys. Lett. B275 (1991)335 .

[6] D. Birmingham, M. Rakowski and G. Thompson, Nucl. Phys. B329 (1990) 83; D. Birmingham and M. Rakowski Mod. Phys. Lett. A4 (1989) 1753; F. Delduc, F. Gieres and S.P. Sorella Phys. Lett. B225 (1989) 367.

[7] S. Fubini and E. Rabinovici Nucl. Phys. B245 (1984) 17; V. de Alfaro, S. Fubini and G. Furlan Nuovo Cimento 34 A, (1976), 569.

[8] E. Witten, Nucl. Phys. B323 (1989) 113.

[9] L. Baulieu, B. Grossman and R. Stora, Phys. Lett. B180 (1986) 95.

[10] A. Forge and E. Rabinovici Phys. Rev. D32, (1985), 927.

[11] A. C. Davis, A. J. Macfarlane, P. C. Popat, and J. W. Van Holten J. Phys. A Math. Gen. 17, (1984), 2945.

[12] E. Witten, Nucl. Phys. B202, (1982), 253. L. Alvarez Gaumé, Comm. of Math. Phys. 90, (1983), 161; D. Friedan and P. Windey Nucl. Phys. B235, (1984), 395.

[13] N.A. Alvez, H. Aratyn and A.H. Zimmerman Phys. Rev. D31, (1985), 3298; R. Akhoury and A. Comtet Nucl. Phys. B246, (1984), 253. 